\title{
Studying the first galaxies with ALMA
}

\author{
C.L. Carilli · F. Walter $\cdot$ R. Wang • A. Wootten • \\ K. Menten - F. Bertoldi - E. Schinnerer - P. Cox · \\ A. Beelen - A. Omont
}

Received: 7 May 2007 / Accepted: 26 July 2007 / Published online: 9 November 2007

(C) Springer Science+Business Media B.V. 2007

\begin{abstract}
We discuss observations of the first galaxies, within cosmic reionization, at centimeter and millimeter wavelengths. We present a summary of current observations of the host galaxies of the most distant QSOs $(z \sim 6)$. These observations reveal the gas, dust, and star formation in the host galaxies on kpc-scales. These data imply an enriched ISM in the QSO host galaxies within 1 Gyr of the big bang, and are consistent with models of coeval supermassive black hole and spheroidal galaxy formation in major mergers at high redshift. Current instruments are limited to studying truly pathologic objects at these redshifts, meaning hyperluminous infrared galaxies $\left(L_{F I R} \sim 10^{13} L_{\odot}\right)$. ALMA will provide the one to two orders of magnitude improvement in millimeter astronomy required to study normal star forming galaxies (i.e. Ly- $\alpha$ emitters) at $z \sim 6$. ALMA will reveal, at sub-kpc spatial resolution, the thermal gas and dust- the fundamental fuel for star formation-in galaxies into cosmic reionization.
\end{abstract}

C.L. Carilli $(\bowtie) \cdot$ R. Wang $\cdot$ A. Wootten

National Radio Astronomy Observatory, 1003 Lopezville Road,

Socorro, N 87801, USA

e-mail: ccarilli@nrao.edu

F. Walter $\cdot$ E. Schinnerer

Max Planck Insitut für Astronomie, Heidelberg, Germany

K. Menten · A. Beelen

Max-Planck Institute for Radio Astronomy, Bonn, Germany

F. Bertoldi

Bonn University, Bonn, Germany

P. Cox

IRAM, Grenoble, France

\section{A. Omont}

Institute de Astrophysique, Paris, France
Keywords Radio astronomy · Extragalactic astronomy · Cosmology $\cdot$ Galaxy evolution and formation

\section{Introduction: Cosmic reionization and the first galaxies}

Observations of the first generation of galaxies and supermassive black holes (SMBH) provide the greatest leverage into theories of cosmic structure formation, and are a principle science driver for all future large area telescopes, from meter to X-ray wavelengths. The recent discovery of the Gunn-Peterson effect, i.e. Ly- $\alpha$ absorption by a partially neutral intergalactic medium (IGM), toward the most distant $(z \sim 6)$ QSOs indicates that we have finally probed into the near-edge of cosmic reionization (Fan et al. 2006a). Reionization sets a fundamental benchmark in cosmic structure formation, indicating the formation of the first luminous objects which act to reionize the IGM. Detection of large scale polarization of the $\mathrm{CMB}$, corresponding to Thomson scattering of the CMB by the IGM during reionization, suggests a significant ionization fraction extending to $z \sim 11 \pm 3$ (Page et al. 2006). Overall, current data indicate that cosmic reionization is a complex process, with significant variance in space and time (Fan et al. 2006b). The on-set of GunnPeterson absorption at $z \geq 6$ implies that the IGM becomes opaque at observed wavelengths $\leq 1 \mu \mathrm{m}$, such that observations of the first luminous objects will be limited to radio through near IR wavelengths.

In this contribution we will discuss the current status of centimeter and millimeter observations of the most distant objects. We will then discuss the revolution afforded by ALMA in this area of research, taking the field from the current study of truly pathologic, rare objects, to the study of the first generation of normal star forming galaxies. In this 


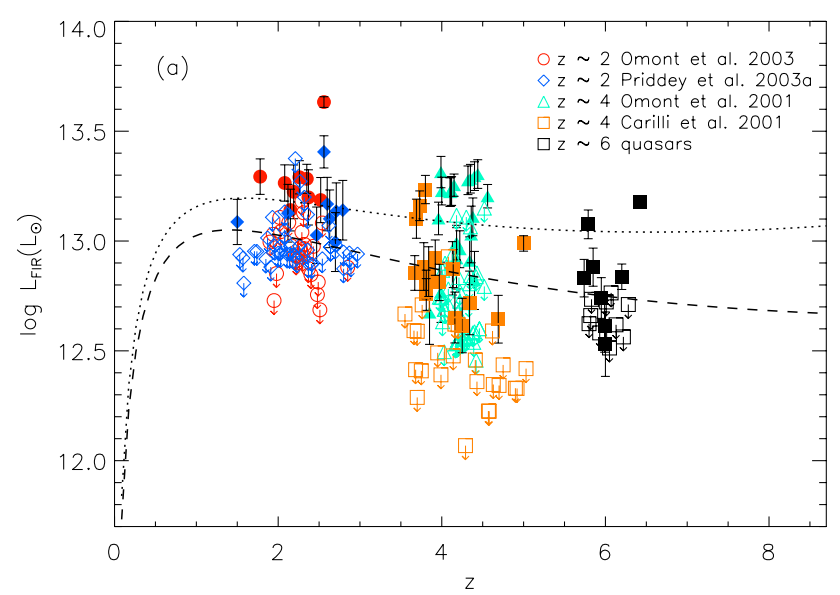

Fig. 1 The logarithm of the FIR luminosity versus redshift for different QSO samples observed at (sub)mm wavelengths (Wang et al. 2007). The open symbols with arrows denote upper limits. The dashed and dotted lines represent the typical $3 \sigma$ detection limits of MAMBO at $250 \mathrm{GHz}$ and SCUBA at $350 \mathrm{GHz}$, namely $S_{250}=2.4 \mathrm{mJy}$, and $S_{350}=8.5 \mathrm{mJy}$, respectively

contribution we concentrate on molecular line and dust continuum emission. Walter and Carilli (this volume) discuss the exciting prospects for studying the fine structure PDR cooling lines, such as [C(II)], from the first galaxies.

\section{Current centimeter and millimeter observations of $z \sim 6$ objects}

\subsection{The host galaxies of $z \sim 6$ SDSS QSOs}

Given the sensitivities of current instruments, observations of sources at $z \sim 6$ are restricted to Hyper Luminous Infrared galaxies (i.e. $L_{F I R}>10^{13} L_{\odot}$ ). At these extreme redshifts, the samples remain limited to the host galaxies of optically luminous QSOs selected from the SDSS. The study of such systems has become paramount since the discovery of the bulge mass - black hole mass correlation in nearby galaxies, a result which suggests a fundamental relationship between black hole and spheroidal galaxy formation (Gebhardt et al. 2000). Our millimeter surveys of the $z \sim 6$ QSOs show that roughly $1 / 3$ of optically selected QSOs are also hyper-luminous infrared galaxies $\left(L_{F I R} \geq 10^{13} L_{\odot}\right)$, emitting copious thermal radiation from warm dust (Fig. 1; Wang et al. 2007). This corresponds to roughly $10 \%$ of the bolometric luminosity of the QSO (which is dominated by the AGN 'big blue bump'), and the question remains open as to the dominant dust heating mechanism: star formation or the AGN?

The best studied of the $z \sim 6$ QSOs is the most distant QSO known, J1148+5251, at $z=6.419$. This galaxy been detected in thermal dust, non-thermal radio continuum, and CO line emission (Walter et al. 2003; Bertoldi et

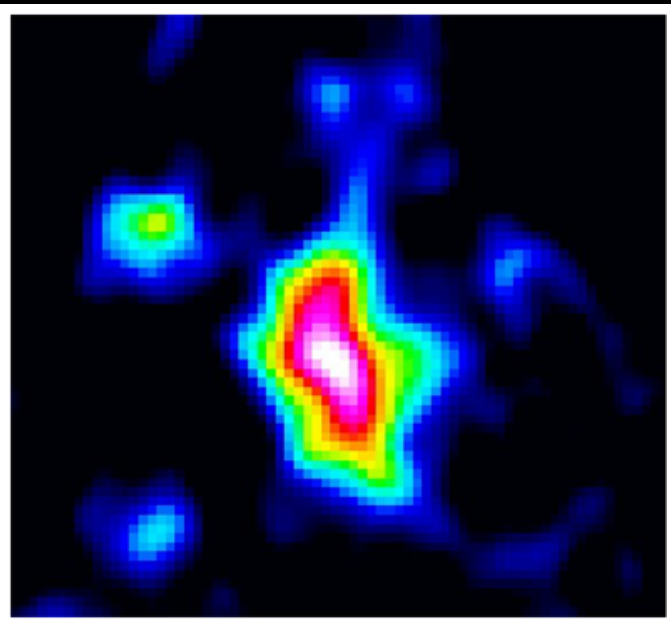

Fig. 2 The velocity integrated CO 3-2 emission from J1148+5251 imaged by the VLA at $0.4^{\prime \prime}$ resolution (Walter et al. 2004). The total flux is $0.15 \mathrm{Jy} \mathrm{km} \mathrm{s}^{-1}$, and the source is clearly resolved, with a full extend of about $1^{\prime \prime}$. The figure is about $3^{\prime \prime}$ on a side

al. 2003; Carilli et al. 2004), with an implied dust mass of $7 \times 10^{8} M_{\odot}$, and a molecular gas mass of $2 \times 10^{10} M_{\odot}$. The molecular gas is extended over $\sim 1^{\prime \prime}$, or $\sim 5.5 \mathrm{kpc}$ (Fig. 2). High resolution VLA imaging of the molecular gas distribution in $\mathrm{J} 1148+5251$ provides the only direct measure of the host galaxy dynamical mass, resulting in a value of $\sim 4 \times 10^{10} M_{\odot}$ within $3 \mathrm{kpc}$ of the galaxy center (Walter et al. 2004; Walter and Carilli, this volume). This mass is comparable to the gas mass, suggesting a baryon-dominated potential for the inner few kpc of the galaxy (Lintott et al. 2006), as is true in nearby spheroidal galaxies, and for ULIRGs (Downes and Solomon 1998). The dynamical mass is also more than an order of magnitude lower than expected based on the bulge mass - black hole mass correlation, suggesting a departure from this fundamental relationship at the highest redshifts, with the SMBH forming prior to the spheroidal galaxy (Walter et al. 2004).

The radio through near-IR SED of J1148+5251 is shown in Fig. 3 (Beelen et al. 2006). The Spitzer bands are consistent with the standard QSO optical through mid-IR SED, including a hot dust component $(\sim 1000 \mathrm{~K})$, presumably heated by the AGN. However, the observed (sub)mm data reveal a clear rest-frame FIR excess. The rest frame FIR through radio SED is reasonably fit by a template that follows the radio through FIR correlation for star forming galaxies (Yun et al. 2000), with a dust temperature of $55 \mathrm{~K}$. The implied star formation rate is of order $3000 M_{\odot}$ year $^{-1}$ (Bertoldi et al. 2003). Most recently, IRAM $30 \mathrm{~m}$ observations of $\mathrm{J} 1148+5251$ have yielded the first detection of the fine structure line of [C(II)] at cosmologically significant redshifts (Maiolino et al. 2005). This line is thought to dominate ISM cooling in photon-dominated regions, i.e. the interface regions between giant molecular clouds and $\mathrm{H}$ (II) regions. 


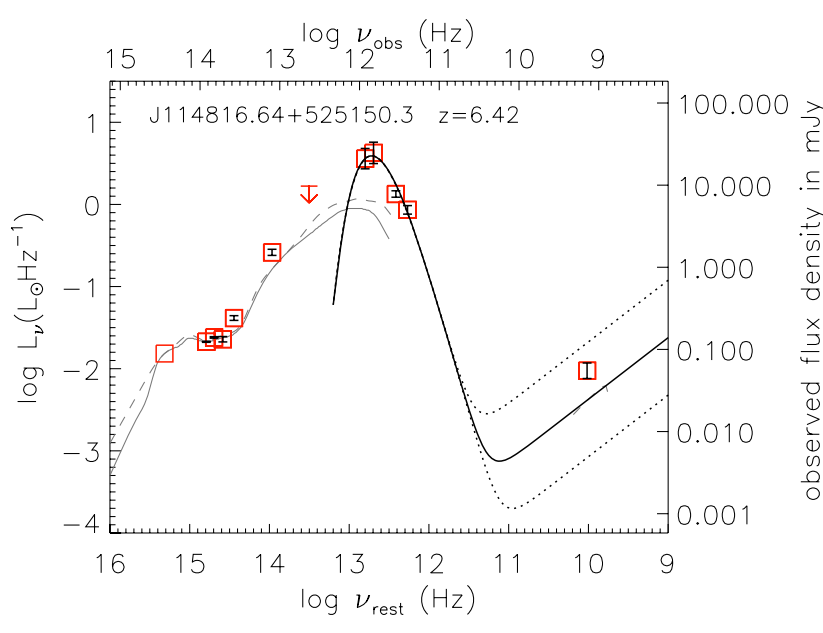

Fig. 3 The SED of J1148+5251 from the rest frame near-IR to the radio (Jiang et al. 2006; Wang et al. 2007; Beelen et al. 2006). The models at rest frame frequencies, $v>10^{13} \mathrm{~Hz}$ are two standard QSO SEDs including emission from hot $(\sim 1000 \mathrm{~K})$ dust. The rest-frame far-IR through radio model entails a $55 \mathrm{~K}$ modified black body, plus synchrotron radio emission that follows the radio-FIR correlation for star forming galaxies (Beelen et al. 2006; Wang et al. 2007). The dashed lines indicate the range defined by star forming galaxies (Yun et al. 2000)

These observations of J1148+5251 demonstrate that large reservoirs of dust and metal enriched atomic and molecular gas can exist in the most distant galaxies, within $870 \mathrm{Myr}$ of the big bang. The molecular gas and [C(II)] emission suggest a substantially enriched ISM on kpcscales. The molecular gas represents the requisite fuel for star formation.

The mere existence of such a large dust mass so early in the universe raises the interesting question: how does a galaxy form so much dust so early in the universe? The standard mechanism of dust formation in the cool winds from low mass (AGB) stars takes a factor two or so too long. Maiolino et al. (2004) and Strata et al. (2007) suggest dust formation associated with massive stars in these distant galaxies. They show that the reddening toward the most distant objects is consistent with different dust properties (i.e. silicates and amorphous carbon grains), as expected for dust formed in type-II SNe (although cf. Venkatesan et al. 2006).

Overall, we conclude that $\mathrm{J} 1148+5251$ is a likely candidate for the co-eval formation of a SMBH through Eddington-limited accretion, and a large spheroidal galaxy in a spectacular starburst, within $1 \mathrm{Gyr}$ of the big bang. This conclusion is consistent with the general notion of 'downsizing' in both galaxy and supermassive black hole formation (Cowie et al. 1996; Heckman et al. 2004), meaning that the most massive black holes $\left(>10^{9} M_{\odot}\right)$ and galaxies $\left(>10^{12} M_{\odot}\right)$ may form at high redshift in extreme, gas rich mergers/accretion events.

Li et al. (2006) and Robertson et al. (2007) have performed detailed modeling of a system like J1148+5251, in-

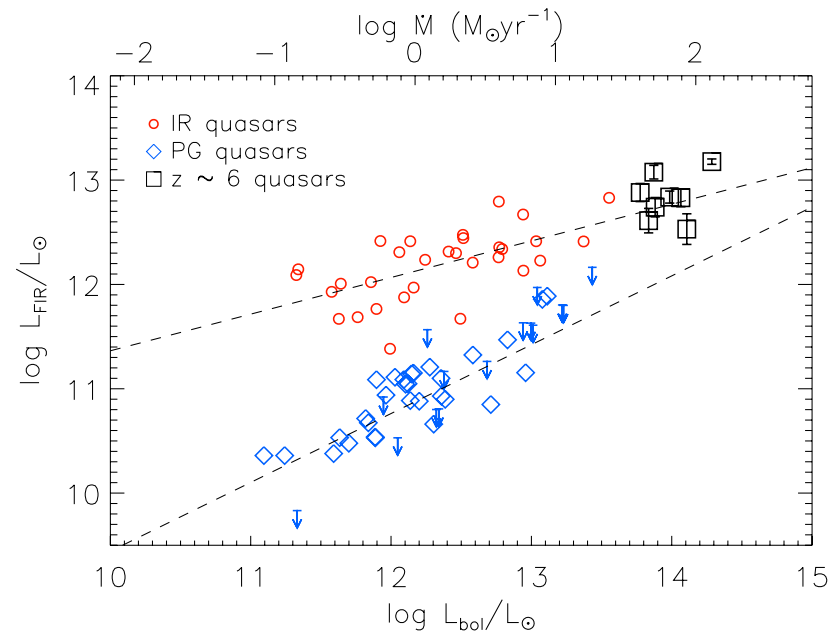

Fig. 4 Correlation of $L_{F I R}-L_{b o l}$ for QSO host galaxies. The (sub)mm detected $z \sim 6$ quasars are plotted as black squares with error bars denoting $1 \sigma$ r.m.s. The local IR and PG quasars from Hao et al. (2005) are plotted as red circles and blue diamonds with arrows denoting upper limits in $L_{\text {FIR }}$. The dashed lines represent the linear regression results for the two local quasar samples

cluding feedback from the AGN to regulate star formation. They show that is plausible to form both the galaxy and the SMBH in rare peaks in the cosmic density field (comoving density $\sim 10^{-9} \mathrm{Mpc}^{-3}$ ), through a series of major mergers of gas rich galaxies, starting at $z \sim 14$, resulting in a SMBH of $\sim 10^{9} M_{\odot}$, and a galaxy of total stellar mass $\sim 10^{12} M_{\odot}$ by $z \sim 6$. The system will eventually evolve into a rare, extreme mass cluster $\left(\sim 10^{15} M_{\odot}\right)$ today. The ISM abundance in the inner few kpc will quickly rise to $\sim$ solar, although the dust formation mechanism remains uncertain.

In support of this conclusion, Fig. 4 shows the relationship between FIR luminosity and bolometric luminosity for the (sub)mm detected $z \sim$ QSOs, as well as a low redshift sample of optically selected QSOs (e.g. PG sample), and an IRAS selected sample of QSOs (Wang et al. 2007; Hao et al. 2005). The $z \sim 6$ sources fall at the extreme luminosity end of the sample, but interestingly, they also follow the trend in FIR to bolometric luminosity set by the low redshift IRAS QSOs, and as opposed to the trend set by the optically selected QSOs. The optically selected QSOs typically have early-type host galaxies, while the IRAS selected QSOs reside in major mergers, with co-eval starbursts (Hao et al. 2005).

A final interesting aspect of the molecular line studies of the most distant QSO host galaxies is the derivation of the sizes of the cosmic Stromgren spheres (Walter et al. 2003; Fan et al. 2006a). The size of the ionized region around the QSO, presumably formed by the radiation from the QSO, can be derived from the difference between the redshift of the host galaxy and the redshift of the on-set of the GunnPeterson trough. For J1148+5251, this redshift difference is 
$\Delta z \sim 0.1$, implying a physical radius for the cosmic Stromgren sphere of $R=4.7 \mathrm{Mpc}$. This radius can be related to the cosmic neutral fraction using the QSO ionizing luminosity, and the mean baryon density, through the equation: $t_{q}=10^{5} R^{3} f(H I)$, where $t_{q}$ is the QSO lifetime, and $f(H I)$ is the IGM neutral fraction (White et al. 2005). For J1148+5251, the implied QSO lifetime is $\sim 10^{7} f(H I)$ years. A number of authors (Wyithe et al. 2005; Fan et al. 2006a; Kurk et al. 2007) have inverted this equation in order to derive the IGM neutral fraction. Using the J1148+5251 CO host galaxy redshift, plus the redshifts for other $z \sim 6$ QSO host galaxies derived from low ionization broad lines (e.g. $\mathrm{Mg}(\mathrm{II})$ ), they derive a mean neutral fraction at $z \sim 6.2$ of $f(H I)>0.1$, assuming a fiducial QSO lifetime $\geq 10^{6}$ years.

\subsection{Limits on normal galaxies: the Cosmos field}

$\mathrm{J} 1148+5251$ is an extremely rare and pathologically luminous object, unlike anything seen nearby. For instance, there are only some 50 or so of these SDSS $z \sim 6$ QSOs on the entire sky!

We have recently investigated the properties of more normal star forming galaxies at $z \sim 6$ using the Ly- $\alpha$ emitting galaxies (LAEs) selected through a wide field, narrow band search of the Cosmos field (Murayama et al. 2007). The sensitivity to the Ly- $\alpha$ line is such that one can detect galaxies with star formation rates of $\sim 10 M_{\odot}$ year $^{-1}$ into cosmic reionization. These galaxies are numerous, with roughly $100 \mathrm{deg}^{-2}$ in a narrow redshift search range of $z=5.7 \pm 0.05$. Extrapolation of the luminosity function to dwarf star forming galaxies could provide enough photons to reionization the universe (Fan et al. 2006a).

We have taken the sample of $\sim 100$ LAEs from the Cosmos field and searched for radio and millimeter emission using MAMBO (Bertoldi et al. 2007) and the VLA (Schinnerer et al. 2007). We do not detect any individual source down to $3 \sigma$ limits of $\sim 30 \mu \mathrm{Jy}_{\text {beam }}{ }^{-1}$ at $1.4 \mathrm{GHz}$, nor do we detect a source in a stacking analysis, to a $2 \sigma$ limit of $2.5 \mu \mathrm{Jy}_{\text {beam }}{ }^{-1}$ (Carilli et al. 2007). At $250 \mathrm{GHz}$ we do not detect any of the 10 LAEs that are located within the central regions of the COSMOS field covered by MAMBO $\left(20^{\prime} \times 20^{\prime}\right)$ to a typical $2 \sigma$ limit of $S_{250}<2 \mathrm{mJy}$. The radio data imply that there are no low luminosity radio AGN with $L_{1.4}>6 \times 10^{24} \mathrm{~W} \mathrm{~Hz}^{-1}$ in the LAE sample.

These radio and millimeter observations rule out any highly obscured, extreme starbursts in the sample, i.e. any galaxies with massive star formation rates $>1500 M_{\odot}$ year $^{-1}$ in the full sample (based on the radio data), or $500 M_{\odot}$ year $^{-1}$ for the $10 \%$ of the LAE sample that fall in the central MAMBO field. The stacking analysis implies an upper limit to the mean massive star formation rate of $\sim 100 M_{\odot}$ year $^{-1}$.
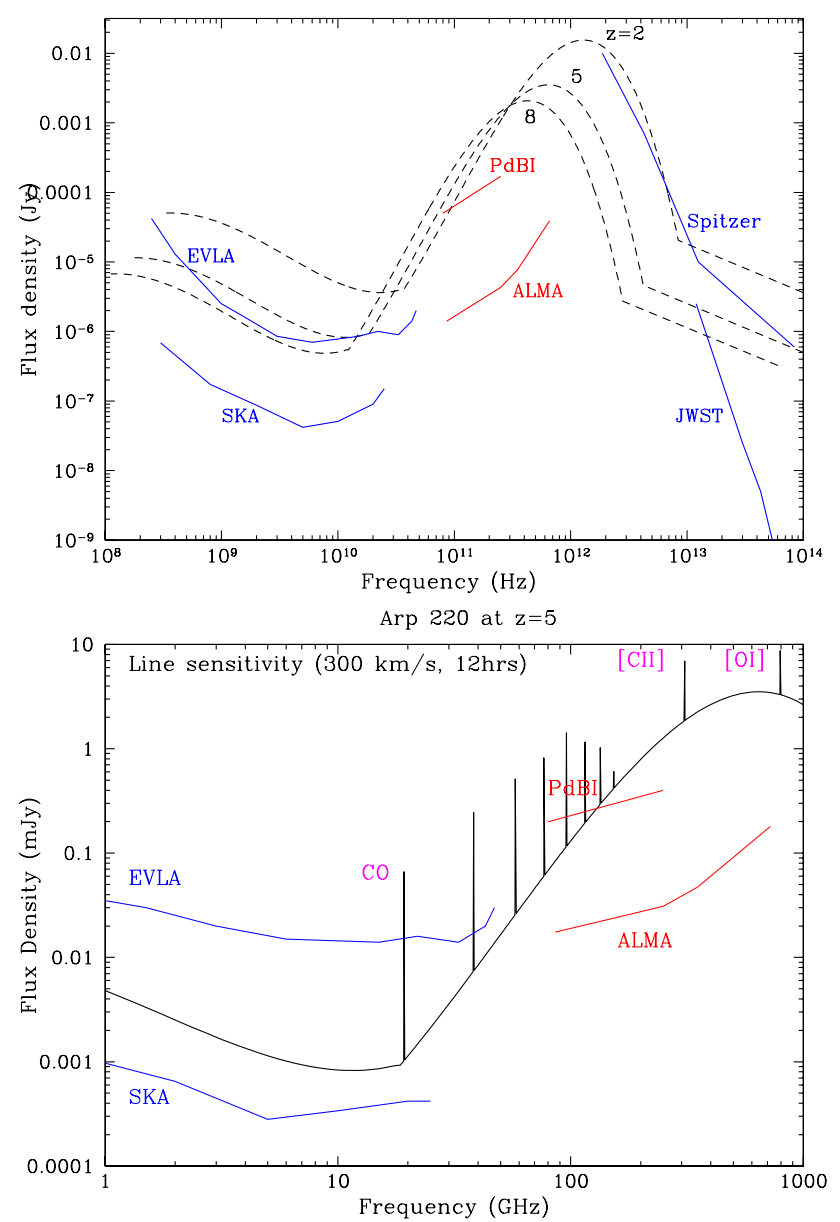

Fig. 5 Top: Continuum spectrum of an active star forming galaxy with a star formation rate $\sim 100 M_{\odot}$ year $^{-1}$, at $z=2,5$, and 8 . The curves show the continuum sensitivities of various telescopes in 12 hours. Bottom: Line spectrum of the same galaxy, but only at $z=5$. The line sensitivities were derived assuming a line width of $300 \mathrm{~km} \mathrm{~s}^{-1}$

While this study represents the most sensitive, widest field radio and mm study of $z \sim 6$ LAEs to date, it also accentuates the relatively poor limits that can be reached in the radio and $\mathrm{mm}$ for star forming galaxies at the highest redshifts, when compared to studies using the Ly- $\alpha$ line.

\section{The ALMA revolution}

ALMA will be able to detect the thermal emission from warm dust from a source like $\mathrm{J} 1148+5251$ in 1 second. Moreover, it will detect the more normal galaxy population $\left(S_{250} \sim 20 \mu \mathrm{Jy}\right.$ for SFR $\sim 10 M_{\odot}$ year $^{-1}$ ), in a few hours.

Figure 5 shows the sensitivity of current and future telescopes from the radio through the near-IR, along with the spectrum of an active star forming galaxy, like Arp 220 (SFR $\sim 100 M_{\odot}$ year $^{-1}$ or $L_{F I R} \sim 10^{12} L_{\odot}$ ). The EVLA, and eventually the SKA, will study the non-thermal (and 


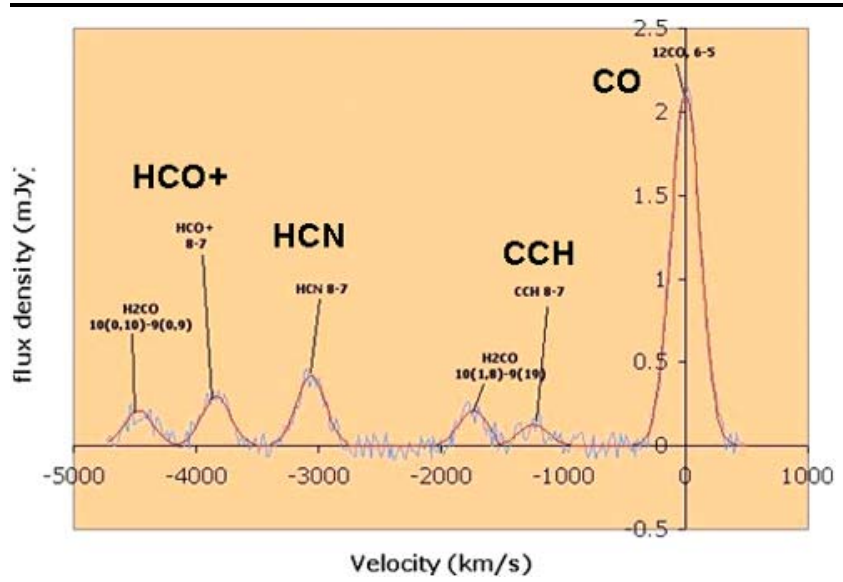

Fig. 6 A simulation of an ALMA spectrum of J1148+5251 at $z=6.42$. The spectrum shows the $8 \mathrm{GHz}$ bandpass, with an integration time of 24 hours, centered around $93 \mathrm{GHz}$

possibly free-free thermal) emission associated with star formation, and possibly AGN, from these distant galaxies, as well as the low order transitions from molecular gas. The JWST will observe the stars, the AGN, and the ionized gas. ALMA reveals the thermal emission from dust and gas, including high order molecular line transitions, and fine structure ISM cooling lines (Walter and Carilli, this volume), from the first galaxies- the basic fuel for galaxy formation. ALMA provides the more than an order of magnitude increase in sensitivity and resolution to both detect, and image at sub-kpc resolution, the gas and dust in normal star forming galaxies (e.g. Ly- $\alpha$ galaxies, with star formation rates $\sim 10 M_{\odot}$ year $^{-1}$ ) back to the first generation of galaxies during cosmic reionization.

As an example, Fig. 6 shows the calculated spectrum for J1148+5251 for the $90 \mathrm{GHz}$ band of ALMA in 24 hours. The $\mathrm{CO}$ lines will be detected with essentially infinite signalto-noise, allowing detailed imaging and dynamical studies on sub-kpc scales. Moreover, in a given $8 \mathrm{GHz}$ bandwidth for ALMA, we will detect transitions from numerous astrochemically interesting molecules, such as the dense, pre-star forming gas tracers, $\mathrm{HCN}$ and $\mathrm{HCO}^{+}$(Gao et al. 2007).

Galaxy formation is a complex process, and proper studies require a panchromatic approach. ALMA represents the more than an order of magnitude increase in sensitivity required to probe normal galaxies into cosmic reionization.

Acknowledgements We acknowledge support from the Max-Planck Society and the Alexander von Humboldt Foundation through the MaxPlanck Forschungspreis 2005. The National Radio Astronomy Observatory is a facility of the National Science Foundation, operated by Associated Universities, Inc.

\section{References}

Bertoldi, F., Carilli, C.L., Cox, P., Fan, X., et al.: Astron. Astrophys. 406, L55 (2003)

Bertoldi, F., et al.: Astrophys. J. (2007, in press)

Beelen, A., et al.: Astrophys. J. 642, 694 (2006)

Carilli, C., et al.: Astron. J. 128, 997 (2004)

Carilli, C., et al.: Astrophys. J. (2007, in press), astro-ph/0612346

Cowie, L.L., et al.: Astron. J. 112, 839 (1996)

Downes, D., Solomon, P.: Astrophys. J. 507, 615 (1998)

Fan, X., et al.: Astron. J. 132, 117 (2006a)

Fan, X., Carilli, C.L., Keating, B.: Annu. Rev. Astron. Astrophys. 44, 415 (2006b)

Gao, Y., et al.: Astrophys. J. (2007, in press), astro-ph/703548

Gebhardt, K., et al.: Astrophys. J. 543, L5 (2000)

Hao, C.N., Xia, X.Y., Mao, S., et al.: Astrophys. J. 625, 78 (2005)

Heckman, T., et al.: Astrophys. J. 613, 109 (2004)

Jiang, L., et al.: Astron. J. 132, 2127 (2006)

Kurk, J., et al.: Astron. Astrophys. (2007, submitted)

Li, Y., et al.: Astrophys. J. (2006, in press), astro-ph/0608190

Lintott, C., Ferreras, I., Lahav, O.: Astrophys. J. 648, 826 (2006)

Maiolino, R., et al.: Nature 431, 533 (2004)

Maiolino, R., et al.: Astron. Astrophys. 440, L51 (2005)

Murayama, T., Taniguchi, Y., Scoville, N.Z., et al.: Astrophys. J. Suppl. Ser. (2007), astro-ph/0702458

Page, L., et al.: Astrophys. J. (2006, in press), astro-ph/0603450

Robertson, B., et al.: Astrophys. J. (2007, in press), astro-ph/073456

Schinnerer, E., et al.: Astrophys. J. (2007, in press), astro-ph/0612314

Strata, G., et al.: Astrophys. J. Lett. (2007, submitted), astro-ph/ 0703349

Venkatesan, A., Nath, B., Shull, M.: Astrophys. J. 640, 31 (2006)

Walter, F., Bertoldi, F., Carilli, C.L., et al.: Nature 424, 406 (2003)

Walter, F., Carilli, C., Bertoldi, F., et al.: Astrophys. J. 615, L17 (2004)

Wang, R., et al.: Astrophys. J. (2007, submitted)

White, R., et al.: Astron. J. 129, 2102 (2005)

Wyithe, S., Loeb, A., Carilli, C.: Astrophys. J. 628, 575 (2005)

Yun, M.S., et al.: Astrophys. J. 528, 171 (2000) 